\title{
ERP-Auswahl für ein Professional Services Unternehmen
}

\section{Norbert Gronau und Malte Teichmann}

Die Branche der Dienstleistungsunternehmen (Professional Services) hat einige Anforderungen, die sie von den "klassischen" ERP-Branchen Industrie und Handel unterscheidet. Dieser Beitrag beschreibt einige der aktuellen Herausforderungen dieses immer wichtiger werdenden Wirtschaftszweigs und geht dann am Beispiel eines mittelständischen Ingenieurdienstleisters auf typische Anforderungen dieser Branche, infrage kommende Systeme und das Vorgehen zur Auswahl ein.

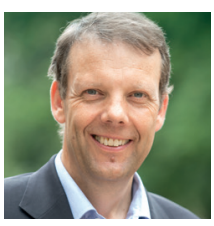

Univ.-Prof. Dr.-Ing. Norbert Gronau ist Inhaber des Lehrstuhls für Wirtschaftsinformatik, insb. Prozesse und Systeme, an der Universität Potsdam.

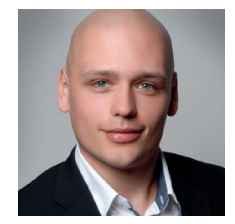

M. A. Malte Teichmann arbeitet als wissenschaftlicher Mitarbeiter am Lehrstuhl für Wirtschaftsinformatik, insb. Prozesse und Systeme an der Universität Potsdam. Zudem ist er als Consultant bei Potsdam Consulting tätig.
$\mathrm{E}$ ine empirische Erhebung des Center for Enterprise Research der Universität Potsdam bei Professional Services-Organisationen zeigt einige aktuelle Trends und Anforderungen im Bereich der betrieblichen Anwendungssysteme. Die befragten Unternehmen verwenden die in Bild 1 gezeigten Ressourcen. Es wird deutlich, dass eine weite Durchdringung nur bei Finanzen, Kontakten und Mitarbeitern gegeben

- wie der aktuelle Stand des ERPEinsatzes in der Branche Professional Services Organisationen ist,

- welche spezifischen Anforderungen an ein ERP-System in Professional Services Organisationen bestehen,

- wie die Auswahl eines ERP-Systems in einer Professional Services Organisation realisiert werden kann. ist, während Material, Kapazität und Management-Information in nicht einmal der Hälfte der Systeme der befragten Unternehmen abgebildet werden.

Der Funnel-to-Cash-Prozess betrachtet den gesamten Serviceprozess von der Erhebung von Anfragen beim potenziellen Kunden bis hin zur Bezahlung. Einer der Ansatzpunkte für die Durchführung der Umfrage lag in der Vermutung, dass die bei den Professional Services-Organisationen eingesetzten Systeme diesen Prozess nicht integriert abbilden. In der Tat haben nur $12 \%$ der befragten Unternehmen einen integrierten Funnelto-Cash-Prozess, während $64 \%$ nicht einmal über teilweise integrierte Systeme verfügen. Dieser niedrige Inte-

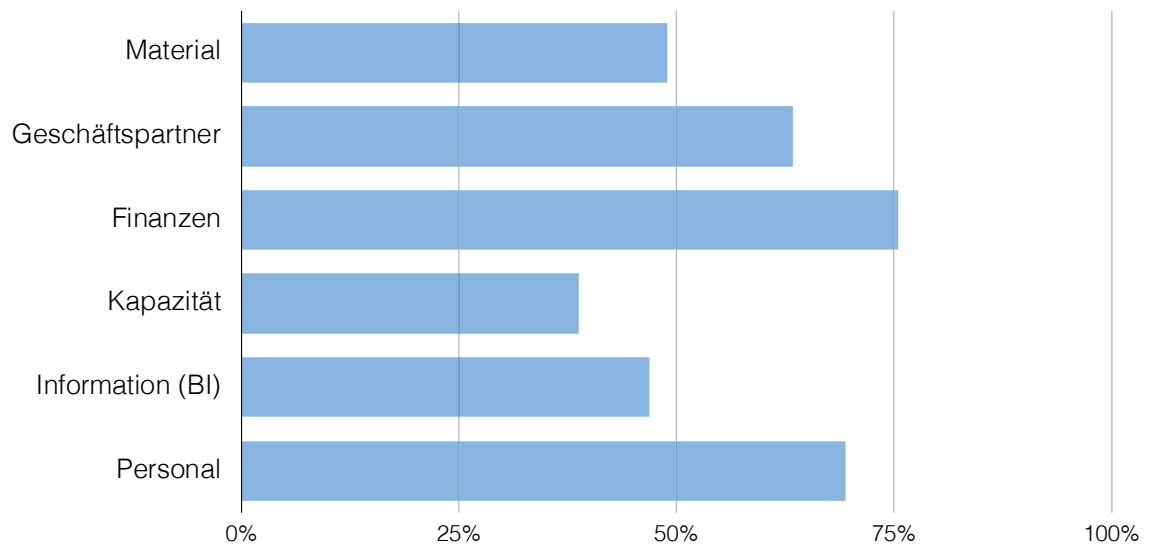

Bild 1: Genutzte ERP-Ressourcen. 
grationsgrad in der Professional Services-Branche deutet darauf hin, dass die Bedeutung eines integrierten Prozesses nicht vollständig erkannt wird, insbesondere, wenn nur vom ERPSystem als möglichem Integrationsanker ausgegangen wird. Immerhin $54 \%$ der beteiligten Unternehmen können bei der Angebotserstellung auf Daten abgeschlossener Projekte zugreifen, wobei mehr als $60 \%$ der Unternehmen detaillierte Angebotsdaten aus abgeschlossenen Projekten verwenden können, nur etwa 30 $\%$ müssen sich mit einer Grobplanung des Projektes begnügen. Diese Funktion, bei der Angebotserstellung auf Daten abgeschlossener Projekte zugreifen zu können, ist für $65 \%$ der befragten Unternehmen wichtig oder sehr wichtig.

Während immerhin noch die Hälfte der Unternehmen die Steuerung des Projektablaufs in ihr System integriert haben, kann auf qualifizierte Ressourcen, auf Bedarfe und auf Bestellungen nur unzureichend zurückgegriffen werden.

Ein deutliches Bild gab die Antwort auf die Frage, ob das derzeit eingesetzte System proaktiv auf das
Erreichen von Schwellwerten oder Deadlines aufmerksam macht. Nur ein kleiner Teil der Befragten kann schon über Systeme auf das Erreichen von Deadlines aufmerksam gemacht werden. Bei mehr als 2/3 der befragten Organisationen werden jedoch diese proaktiven Meldungen als wichtig oder sehr wichtig angesehen, so dass auch hier ein klares Delta konstatiert werden kann zwischen dem, was im Einsatz ist, und dem, was wünschenswert ist.

Nur $14 \%$ der befragten Organisationen nutzen gegenwärtig bereits ein Tool zur Professional Services Automation. Solche Werkzeuge liefern eine Systembasis für die Initiierung und Planung des Ressourcenmanagements, die Ressourceneinteilung, die Durchführung, den Abschluss und die Steuerung von Projekten und Dienstleistungen.

In PSA-Tools ist ein Dashboard für Ressourcen und Projekttermine enthalten, das auch eine Funktion zur Vorhersage der Nachfrage enthält. Derartige Werkzeuge helfen, die Durchführung der Services zu verwalten. PSA-Tools verfügen über Funktionen zum Einplanen von Ser-
vice-Opportunities, zur Zusammenstellung von Serviceteams und über Werkzeuge für die Zusammenarbeit im Projekt einschließlich der Terminund Ressourcenplanung. Ein PSA-Tool ist typischerweise dasjenige System, in dem die Fähigkeiten der Mitarbeiter, ihre Kompetenzen und Präferenzen aufgezeichnet werden. Es wird darüber hinaus benutzt, um Zeiten, Aufwendungen und Ressourcen bis auf Aufgabenniveau herunter zu erfassen. Daher wird es auch als Basis für die Erstellung von Projektkostenschätzungen verwendet. Die meisten PSA-Tools bieten auch Rechnungsmodule an, die unterschiedliche Abrechnungsarten unterstützen (Time and Materials, Teilzahlungen oder Festpreisprojekte). Der zukünftige Einsatz eines Professional Services Automation Tools wird überraschenderweise nur von einem Viertel der befragten Unternehmen als wichtig oder sehr wichtig eingestuft.

Elektronische Rechnungsformate werden bereits in den Unternehmen genutzt, haben sich aber noch nicht flächendeckend durchgesetzt. Mobile Anwendungen sind den Unternehmen zu gleichen Teilen wichtig und weniger
Identifizierte spezifische Herausforderungen

Die Organisation von Projekten (Start/Enddatum, Ressourcen etc.) ist einzelfallabhängig und kann je nach Projektziel stark variieren

Der Funnel-to-cash-Prozess wird administrativ oft durch einzelne Projektleiter übernommen

(Ausbleibende) Wertschöpfung in der Projektarbeit ist durch fehlende Transparenz nicht immer erkennbar

Effektive Projektarbeit ist von einer ressourcen- und zeiteffizienten Aufgabenverteilung abhängig

Arbeitspakete hängen oft von der Erfüllung vorgelagerter Aufgaben ab

Branchen- und Auftraggeber spezifische Spezifika (z.B. bei Honorarverordnungen, Dateiformaten)

Projektarbeit erfordert stetig wiederkehrende sowie variierende materielle und immaterielle Güter
Spezifische Anforderungen an das ERP-System

Die Organisation von Projekten muss flexibel abbildbar sein, wobei sich einzelne Aufgabenpakete, Meilensteine etc. aus dem Projektangebot ergeben können

Eine hohe Datenintegrität von der Projektanbahnung bis -abrechnung und -controlling ist obligatorisch

Etablierung und Vertiefung des Verständnisses für wertschöpfende Prozesse durch Transparenz auf allen Ebenen des Unternehmens (z.B. KPI für verschiedene Stakeholder)

Verplante und verfügbare personelle Ressourcen müssen leicht abbild- bzw. einplanbar sein

Automatische Benachrichtigungen bei Aufgabenerfüllung müssen (z.B. durch Workflows) gegeben sein

Abbildung und Bereitstellung von Schnittstellen gängiger Brancheninstrumente

Flexible Beauftragung und Einplanung externer und interner Dienstleistungen im Projektplan 
wichtig. Ein Webportal nutzt derzeit nur ein Viertel der befragten Unternehmen, wohingegen eine weitere Hälfte einzelne Anbindungen von Kunden oder Partnern bereits realisiert hat.

Die Umfrage zeigt, dass in der Branche noch erhebliche Lücken in der Prozessabdeckung mit geeigneten Informationssystemen existieren. Gleichermaßen gestaltet sich der Auswahlprozess aufgrund der Spezifika von Professional Services Unternehmen als schwierig.

\section{ERP-Anforderungen: Ingenieurdienstleister}

Der hier vorgestellte Ingenieurdienstleister ist in den letzten zehn Jahren sehr stark gewachsen und konnte seinen Umsatz auf einen zweistelligen Millionenbetrag steigern. Die Tätigkeit des Unternehmens besteht aus Ingenieurdienstleistungen, die mit rund 150 erfahrenen Ingenieuren sowie technischen Mitarbeitern erbracht werden, die von unterschiedlichen Standorten in Deutsch- land aus agieren. Die geschäftsführenden Gesellschafter gehen davon aus, dass das Unternehmen in den nächsten Jahren weiter wachsen wird. Derzeit werden alle wertschöpfenden Prozesse mit Hilfe von Bürosoftware (Word, Excel, Access) abgebildet. Die Buchhaltung erfolgt über DATEV online. Die gegenwärtigen Prozesse sind durch ein hohes Maß an Intransparenz, z. B. über Profitabilität der einzelnen Projekte, Verfolgung von Nachträgen und Abrechnung von Leistungen gekennzeichnet. Wesentliche Ist-Prozesse sind in Form von Qualitätsdokumenten dokumentiert. Ebenfalls fehlt ein Überblick über den fachlichen Bearbeitungsstand von Projekten. Ein Einblick in die reale Auslastung von Personal ist damit nur schwer bzw. eine davon abhängige Ressourcenplanung nur rudimentär möglich.

Unter Zuhilfenahme externer Berater wurde eine zukunftsfähige Systemlösung ausgewählt und eingeführt. Der Prozess der Systemauswahl im Sinne eines adaptierten Vorgehens
[1] orientierte sich am Software-Auswahlverfahren, dass von Potsdam Consulting in Zusammenarbeit mit dem an der Universität Potsdam beheimateten Center for Enterprise Research erarbeitet und in einer Vielzahl von Praxisprojekten validiert worden ist [2]. Über den gesamten Auswahlprozess wurde ein besonderer Fokus auf die Spezifika des Wirtschaftszweiges Professional Services Unternehmen gelegt. Tabelle 1 stellt wesentliche Herausforderungen und daraus resultierende Anforderungen an die ERP-Auswahl aggregiert dar:

\section{Auswahlverfahren der Systemlösung}

Im Folgenden wird das Auswahlverfahren unter Berücksichtigung der Anforderungen skizziert.

\section{Anforderungserhebung}

Auf der Basis einer vom Management gelieferten Zieldefinition wurden grundlegende Anforderungen an das neue ERP-System ermittelt. Daran

\begin{tabular}{|c|c|}
\hline Lfd. Nr. & Anforderung \\
\hline 1 & $\begin{array}{l}\text { Besteht die Möglichkeit, relevante Daten (Anzahl, wirtschaftlicher Status, letzte Abrechnung, offene Stunden, offene } \\
\text { Leistungen etc.) von Projekten der Niederlassungen automatisch ausweisen zu lassen? }\end{array}$ \\
\hline 5 & Sind GAEB-Daten in Ihrem System einles- und schreibbar? \\
\hline 10 & Ihr System unterstützt die automatische Erstellung eines Projektplanes aus dem Angebot heraus? \\
\hline 11 & Die Aufgliederung von Projekten in einzelne Arbeitspakete, strukturierte Aufgaben und Meilensteine ist möglich? \\
\hline 20 & $\begin{array}{l}\text { Mitarbeiter werden automatisch benachrichtigt, wenn bestimmte Ereignisse (z. B. Aufgabenerfüllung) die Bearbei- } \\
\text { tung eigener Aufgaben ermöglichen? }\end{array}$ \\
\hline 22 & $\begin{array}{l}\text { Aufgaben können durch den Projektleiter einzelnen Projektbearbeitern zugewiesen werden, wobei das System eine } \\
\text { automatische Plausibilitätsprüfung (z. B. Wochenarbeitszeit) vornimmt. }\end{array}$ \\
\hline 30 & Existiert in Ihrem System ein Auftragsmanagement zur Buchung und Abwicklung interner Dienstleistungen? \\
\hline 34 & Existiert die Möglichkeit, externe Dienstleistungen aus dem System heraus zu beauftragen? \\
\hline 35 & Sind externe Dienstleistungen im Projektplan terminierbar? \\
\hline 60 & $\begin{array}{l}\text { Können die zuständigen Projektleiter automatisch benachrichtigt werden, wenn relevante Daten für die Rechnungs- } \\
\text { erstellung fehlen? }\end{array}$ \\
\hline 61 & $\begin{array}{l}\text { Werden KPIs (z. B. Projektanteil am UN-Umsatz, Gewinnhöhe einzelner Projekte etc.) eines Projektes systematisch } \\
\text { erhoben? }\end{array}$ \\
\hline
\end{tabular}

Tabelle 2: Auflistung beispielhafter Anforderungen eines Ingenieurdienstleisters. 
anschließend wurden Anforderungen der Leitungsebene und einzelner Fachabteilungen mithilfe von Interviews und vorliegenden Unterlagen zum Unternehmen erhoben und in einer ersten Anforderungsliste zusammengefasst. Tabelle 2 zeigt eine vereinfachte Darstellung ausgewählter Anforderungen:

Die vollständige Liste wurde mit dem Auftraggeber diskutiert bzw. alle Anforderungen hinsichtlich Ihrer Priorität gewichtet sowie in einem Anforderungskatalog (inkl. Darstellung der jeweiligen Fachabteilungen, funktionaler Anforderungen etc.) zum Zwecke der Systemselektion dokumentiert.

\section{Marktübersicht und Screening}

Anschließend wurden zur Auswahl einer Topliste aus dem ERPGesamtmarkt ca. 25 infrage kommende Anbieter selektiert und hoch priorisierte Anforderungen abgefragt. Dabei wurde sich sowohl auf branchenspezifische als auch allgemeiner gefasste Systemlösungen im Bereich Professional Services Unternehmen konzentriert (Tabelle 3).

Der Unterschied zu Auswahlprozessen im Bereich Industrie und Handel lag darin, dass insgesamt wesentlich weniger Systeme als infrage kommende Lösungen identifiziert werden konnten. Dies lag an der vergleichsweise geringen Anzahl überhaupt angebotener Lösungen im Bereich projektgesteuerter Dienstleistungen. Weiterführend wurden durch die Vorselektion weitere 19 Systeme ausgeschlossen, da unter anderem Anforderungen an die Projektsteuerung (z.B. automatische Erstellung des Projektarbeitsplanes aus dem Angebot) bzw. branchenspezifische Anforderungen (z.B. Abbildbarkeit von GAEB-Dateien) nicht in ausreichendem Maße adressiert wurden. Im Ergebnis wurde eine Shortlist mit sieben Anbietern erstellt.

\section{Qualifizierung für die Anbieter- präsentationen beim Kunden}

Zur Vorbereitung der Anbieterpräsentationen beim Kunden wurde ein Präsentationsleitfaden für die

\begin{tabular}{ll|l|l|l}
$\begin{array}{l}\text { Anbieter erstellt. } \\
\text { Dieser enthielt } \\
\text { neben der Beschrei- }\end{array}$ & 1 & WECLAPP & 14 & Abacus \\
bung wesentlicher & 2 & Anbieter & Lfd. Nr. & Anbieter \\
$\begin{array}{l}\text { Geschäftsprozesse } \\
\text { ebenfalls charak- }\end{array}$ & 3 & StepAhead & 16 & Kobold \\
teristische Daten & 4 & Oxaion & 17 & HHK Datentechnik \\
erfolgreich absol- & 5 & Godesys & 18 & Projekt Pro \\
vierter Projekte. & 6 & Unit4 & 19 & SAP \\
$\begin{array}{l}\text { Diese orientieren } \\
\text { sich an den hoch }\end{array}$ & 7 & Softsite & 20 & Scopevisio \\
priorisierten Anfor- & 8 & Scholz.MSC & 21 & Deltek \\
derungen. Alle & 9 & Connectivity & 22 & Kumavision \\
Anbieter der Short- & 10 & Work4all & 23 & WIKO \\
list zeigten ihre & 11 & StaWare & 24 & Sonapro \\
vorgesehenen Prä- & 1 & Verteck & 25 & All4cloud \\
sentationen bereits & 12 & Axavia & 26 & Qalgo \\
vorab in Webcasts. & 13 & &
\end{tabular}

Die konsultierten

Berater bewerte-

Tabelle 3: Anbieter aus dem Bereich systemgesteuerter Dienstleistungen.

und erzeugten eine

Einschätzung der Anbieter nach Qualität des Systems und Fähigkeit zur Adaption der identifizierten Anforderungen. Die vier bestgeeignetsten Systeme bzw. deren Vertreter wurden final zu einer Anbieterpräsentation vor Ort eingeladen.

Hier ist auf eine Besonderheit im Auswahlprozess hinzuweisen: Während in "klassischen" Bereichen die jeweiligen Präsentationsleitfäden von WebCast und Vor-Ort-Präsentationen identisch sein können, erfordert das Vorgehen bei Professional Services Unternehmen bzw. dafür auszuwählender Systemlösungen ein gesondertes Vorgehen.

Zwar zeichnen sich Systeme beider Bereiche durch eine starke Interoperabilität der jeweiligen Module aus, die gesonderte Betrachtung einzelner Module gelingt bei ersteren jedoch besser als bei letzteren. Dies ist nicht zuletzt auf die Charakteristika von Projekten zurückzuführen, was insbesondere beim Vergleich der Zusammenwirkung der potenziellen Module Einkauf und Warenwirtschaft vs. Projektanbahnung und Projektplanung deutlich wird. Während etwa Prozesse im Einkauf unabhängig von der späteren Lagerplatzverwaltung betrachtet werden können, ist die Planung von Mei- lensteinen, Aufgaben und Deadlines in einem Projekt stark von den in der Projektanbahnung verhandelten Projektinhalten abhängig. Eine Demonstration von Projektplanungs-Funktionen innerhalb eines Demonstrationssystems ist zwar auch ohne Daten aus der Projektanbahnung möglich, würde jedoch vor dem Hintergrund der Einschätzung der notwendigen Datenintegrität und Funktionsumfänge nachgelagerter Projektschritte (z. B. Verfassen der Rechnung) wenig Sinn ergeben.

Im Ergebnis kann dementsprechend eine chronologische Anpassung des Präsentationsleitfadens an den natürlichen Flow eines Systems sinnvoll sein, wenn die für den Kunden relevanten Funktionsumfänge dadurch besser herausgestellt werden können. Für die objektive Vergleichbarkeit der Systeme ist jedoch obligatorisch, dass auch in diesem Fall alle Anforderungen inhaltlich adressiert werden. Dies sollte deutlich und mit Nachdruck an die ERP-Anbieter kommuniziert werden.

\section{Anbieterpräsentationen und Auswahlvorschlag}

Jeder der zur Präsentation eingeladenen Anbieter zeigte die zur Ent- 
scheidung wesentlichen Abläufe und Funktionen des Systems mit (anonymisierten) Projektdaten. Vier Systeme wurden zur Präsentation eingeladen, wobei die Bewertung sowohl durch Vertreter des Unternehmens als auch der Berater erfolgte. Unter Einbezug der durch die Anbieter vorgelegten kaufmännischen Angebote wurde eine finale Empfehlung für den projektgesteuerten Ingenieurdienstleister gegeben. Die anschließenden Vertragsverhandlungen wurden durch das beratende Unternehmen unterstützt.

\section{Zusammenfassung}

Empirische Untersuchungen zeigen, dass der Wirtschaftszweig der projektgesteuerten Dienstleistungen noch nicht alle Potenziale von ERP-Systemen flächendeckend und in vollem Ausmaß ausschöpft hat. Ebenfalls sind besondere Anforderungen an ERP-Systeme erkennbar, die sich primär aus den Herausforderungen der Projektbearbeitung (z.B. Heterogenität im Rahmen der Projektorganisation) bzw. branchenspezifische Besonderheiten (z. B. Schnittstelle zu besonderen Dateiformaten) ergeben. Sowohl für interessierte Unternehmen als auch neutrale Beratungen kommt ebenfalls die Schwierigkeit hinzu, dass im Vergleich zu den "klassischen" Branchen Industrie und Handel vergleichsweise wenige Anbieter am Markt sind. Der vorliegende Beitrag und der darin skizzierte erfolgreiche Auswahlprozess können als praxisnahe Handreichungen verstanden werden, wie eine ERP-Auswahl unter den aufgezeigten Rahmenbedingungen gelingen kann.
Literatur:

[1] Bender, B., Gronau, N.: Auswahl von ERPSystemen im Kontext von Individuallösungen. Fundort: https://www.erp-management.de/node/1562 (Letzter Zugriff: 30.05.2020)

[2] Gronau, N.: Die Zeit ist reif: Ein neues ERPAuswahlverfahren, ERP Management 2/2014, S. 45-47

Schlüsselwörter

ERP-Systeme, ERP-Auswahl, Auswahlvorgehen, Professional Services Unternehmen

(1)

Kontakt:

Lehrstuhl für Wirtschaftsinformatik, insb. Prozesse und Systeme

Universität Potsdam

Prof. Dr.-Ing. Norbert Gronau

August-Bebel-Straße 89

14482 Potsdam

http://wi.uni-potsdam.de

\section{Das richtige ERP-System}

Auswahlentscheidungen für ein neues ERP-System werden häufig unsystematisch durchgeführt. Die Unsicherheit, ob das richtige System ausgewählt wurde, bleibt groß. Dies behindert den Einführungsprozess.

Das Handbuch der ERP-Auswahl kann Ihnen helfen, das richtige ERP-System auszuwählen. Sie haben unmittelbar Zugriff auf Checklisten, Templates und alle notwendigen Informationen, um die Auswahlsicherheit bei der ERP-Auswahl zu steigern:

- Ein Leitfaden für alle Schritte der ERP-Auswahl

- Checklisten u.a. für Architektur, Betriebsform, Beraterauswahl, Ergonomie

- Templates für klare und präzise Antworten von Anbietern und Beratern

- Fachaufsätze u.v.m.

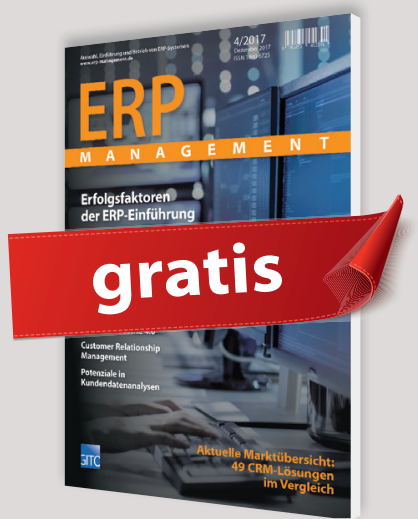

\section{Erfolgsfaktoren zur ERP-Einführung}

mit den Schwerpunktthemen:

- Change Requests

- Fallstudie zur effizienten ERP-Auswahl

- Stammdatenaustausch

- Innovative Digitalisierungsstrategien

- ERP und Industrie 4.0

- Customer Relationship Management

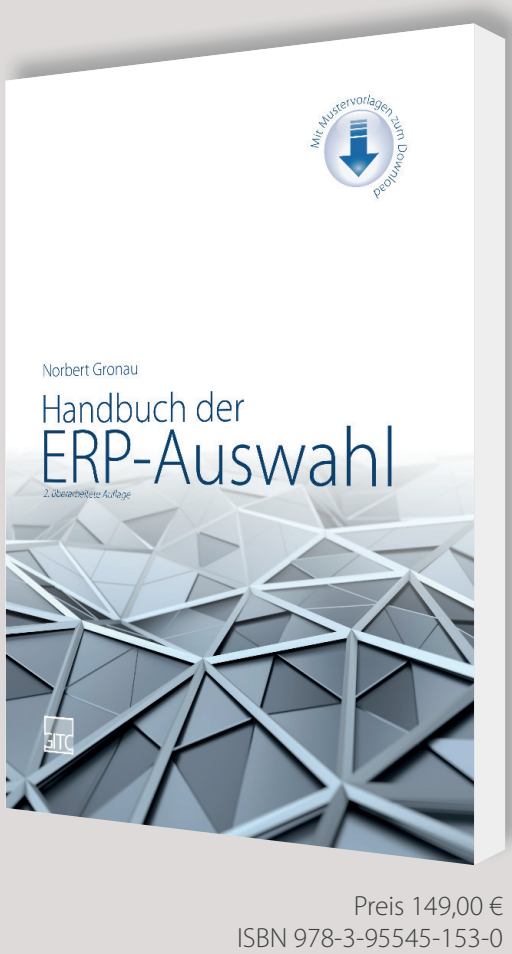

Sie bestellen das Handbuch der ERP-Auswahl und erhalten kostenlos dazu die Erfolgsfaktoren zur ERP-Einführung 